\title{
Existence of Coefficient Subring for Transcendental Extension Ring
}

\author{
Hanan Alolaiyan \\ Mathematics Department, King Saud University, \\ holayan@ksu.edu.sa
}

\section{ABSTRACT}

As a consequence of Cohen's structure Theorem for complete local rings that every finite commutative ring $R$ of characteristic $p^{n}$ contains a unique special primary subring $R_{0}$ satisfying $R / J(R) \cong R_{0} / p R_{0}$. Cohen called $R_{0}$ the coefficient subring of $R$. In this paper we will study the case when the ring is a transcendental extension local artinian duo ring $\mathrm{R}$; we proved that even in this case $R$ will has a commutative coefficient subring.

Mathematics Subject Classification 2010 [MSC]: 13B05, 12F20, $13 \mathrm{H} 99$.

Keywords: Transcendental Extension; Algebraic Extension; Coefficient Subring; Artinian ring.

\section{Introduction}

It is well known that Wedderburn-Mal'tsev Principal Theorem is essential to know the structure of a given ring, many scientists tried to improve it. I.S. Cohen [7] proved that any commutative local notherian ring $\mathrm{R}$ that is $J(R)-$ adic complete have a subring $R_{0}$ such that $R=R_{0}+J(R), R_{0} \cap J(R)=J\left(R_{0}\right)$. On the contrary, Asumaya [5] introduced the concept of an inertial sub algebra of algebra over a Hensel ring. The concept of an inertial sub algebra is analogous to that of a coefficient subring Y. Alkhamees and S. Singh have generalized the result to the existence of coefficient subring of a locally finite $R$-algebraA, where $R$ is a commutative chain ring such that $J(R)=R \cap J(A), A / J(A)$ countably generated separable algebraic field extension. A. Azarang [3] discussed similar concept of coefficient subring which is maximal subring and he showed that the existence of maximal subrings in some commutative noetherian ring. Also, A. Pavelescu [14] proved that maximal commutative subgrings of Artinian semisimple rings are direct products of local rings. As we know that there are many important rings that are not algebraic extension of its prime subring. Actually the existence and structure of $R_{0}$ for finite commutative local ring $R$ was known to Krull as early as 1924 [11] , p.20. For finite commutative local ring it turns out that $R_{0}$ is a Galois ring. Clark [6] proved that a coefficient subring of finite $p-\operatorname{ring} R$ is a direct sum of full matrix rings over Galois rings. Finally Corbas [8] manages to characterize coefficient subring of a finite ring as a direct sum of full matrix rings over Galois rings.Y. Alkhamees and S. Singh [1] have generalized the results on the existence of coefficient subrings of finite local rings to locally finite $R$-algebra $A$, where $R$ is commutative chain ring such that $J(R)=R \cap J(A), A / J(A)$ countably generated separable algebraic field extension. By Cohenâ $€^{\mathrm{TM}} \mathrm{s}$ structure theorem, $R$ is a complete domain as a finite integral extension of a complete local regular domain $R_{0}$, and if $R$ has the similar features as its field of residue, then $R_{0}$ is a recognized series of power ring over a field [7]. J. Ã-inert, J. Richter, and S.D. Silvestrov [13] proved that if $R$ is a commutative domain of characteristic zero. Then its Ore extension $R\left[x ; i d_{R}, \delta\right]$ is a simple ring if and only if $R$ is $\delta$-simple and a maximal commutative subring of $R\left[x ; i d_{R}, \delta\right]$. Moreover, In [13] they considered centralizers of single elements in certain Ore extensions, with a non-invertible endomorphism, of the ring of polynomials in one variable over a field, and showed that they are commutative and finitely generated as an algebra.

The researches above were focused on studying the case when the ring is finite or even algebraic extension over its subring. Though, there are many important non-commutative rings that are not algebraic extension of its prime subring. So in this research, the focus will be on the case when a ring is any transcendental extension of its prime subring. Let $R$ be an artinian local duo ring such that $\bar{R}$ is a field(algebraic and transcendental extension).Some propositions have been given to reach our main theorem which asserts that $R$ has a coefficient subring $R_{0}$, which is either a field or a transcendental extension of a generalized Galois subring $R_{0}$ which is a coefficient subring of the 
ring of all algebraic elements in $R$ over the prime subring.

\section{Preliminaries}

All rings considered in the research have $1 \neq 0$. Let $R$ be any ring. Then the smallest subring $P$ of $R$ containing the identity $1 \neq 0$ such that for any integer $n$, if $n 1$ is a unit in $R$, then $(n 1)^{-1} \in P$, is called the prime subring of $R$. The prime subring of a field $F$ is the prime subfield of $F . J(R), C(R)$ denote the Jacobson radical and center of $R$ respectively. Let $R$ be a commutative local ring and $\bar{R}=R / J(R)$. For any $f(x) \in R[x]$, let $\overline{f(x)}$ denote its natural image in $\bar{R}[x] . R$ is called a Hensel ring, if it satisfies the following condition : Let $f(x) \in R[x]$ be any monic polynomial . If $\bar{f}(x)=g_{0}(x) h_{0}(x)$ for some relatively prime monic polynomials $g_{0}(x), h_{0}(x) \in \bar{R}[x]$, then there exist monic polynomials $g(x), h(x) \in R[x]$ such that $f(x)=g(x) h(x), \bar{g}(x)=g_{0}(x), \bar{h}(x)=h_{0}(x)$. By Hensel lemma any complete local commutative ring is a Hensel ring, in particular any commutative local artinian ring is a Hensel ring. A ring $R$ is called chain ring, if it is a local, both sided artinian, principal ideal ring. A commutative chain ring $R$ is called a special primary ring. A finite special ring $S$ such that $J(S)=p S$, where $p=\operatorname{char}(S / J(S))$ is a Galois ring .A ring $R$ in which each onesided ideals is two sided is called a duo ring. Let $R$ be a local ring, such that $R / J(R)$ is a field. Then a monic polynomial $f(x)=\sum_{i=0}^{r} a_{i} x^{i} \in R[x]$ is said to be partially irreducible [separable] polynomial over $R$, if $f(x)$ is irreducible [separable] modulo $J(R)$, in the sense that for $\bar{R}=R / J(R), \bar{f}(x)=\sum_{i=0}^{r} \overline{a_{i}} x^{i} \in \bar{R}[x]$ is irreducible [separable] over $\bar{R}$. If $f(x)$ partially irreducible polynomial over $R$, then it is easy to see that $\langle f(x)\rangle$ $+J(R)[x]$ is a maximal ideal of $R[x]$. Let $R$ be a commutative, local ring with $J(R)$ nilpotent, and let $P_{0}$ be its prime subring . Let $f(x) \in R[x]$ be a partially irreducible separable polynomial over $R$, such that for some $a \in R, f(x) \equiv(x-a) g(x)(\bmod J(R)[x])$. Then there exist $a_{1} \in R, g_{1}(x) \in R[x]$, such that $a_{1} \equiv a$ $(\bmod J(R)), g_{1}(x) \equiv g(x)(\bmod J(R)[x])$, and $f(x)=\left(x-a_{1}\right) g_{1}(x)$.

Definition 1 Let $R$ be a ring. Then $R$ is called a generalized Galois ring if $R=\cup_{i \in \Lambda} R_{i}$, where all $R_{i}$ are Galois rings of the same characteristic and for any $\alpha, \beta \in \Lambda$ there exists $r \in \Lambda$ such that $R_{\alpha} \cup R_{\beta} \subseteq R_{r}$.

It is easy to see that if $R=\cup_{i \in \Lambda} R_{i}$ is a generalized Galois ring, then $R$ is a local, artinian principle ideal ring.

Definition 2 Let $R$ be a local ring such that $\bar{R}$ is a field and $T$ a commutative local subring of $R$, $J(T)=J(R) \cap T$. Then $(i)$ An element $a$ in $R$ is said to be a lift algebraic element over $T$ if there exists a partially irreducible polynomial $f(x)$ over $T$ such that $f(a)=0 ; f(x)$ is called a minimal polynomial of a over $T$. If deg $f(x)=r$, then $a$ is said to be of degree $r$ over $T$. (ii) A lift algebraic element $a$ in $R$ over $T$ is said to be separable over $T$ if its minimal polynomial over $T$ is separable.

It is well known that for a commutative local ring $T$ with $J(T)$ nilpotent, and $T^{\prime}$ a local subring of $T$ such that $J\left(T^{\prime}\right)=T^{\prime} \cap J(T)$, if $\bar{a} \in \bar{T}$ separable over $\overline{T^{\prime}}=\left(T^{\prime}+J(T)\right) / J(T)$ and $f(x)$ a monic polynomial over $T^{\prime}$ such that $\bar{f}(x)$ the minimal polynomial of $\bar{a}$, then there exist a unique element $b \in \bar{a}$ such that $f(b)=0$. Let $R$ be a commutative artinian local ring with $\bar{R}$ an absolutely algebraic field, and let $(T, \pi)$ be a special primary 
subring of $R$. Suppose that $\bar{a}$ is a separable element in $\bar{R}$ over $\bar{T}=(T+J(R)) / J(R)$ and $f(x)$ a partially irreducible polynomial over $T$ such that $\bar{f}(\bar{a})=0$. Then there exist a unique lift algebraic element $a^{\prime} \in R$ over $T$ such that $f\left(a^{\prime}\right)=0$ and $\overline{a^{\prime}}=\bar{a}$. Then $T\left[a^{\prime}\right]$ is determined by the field $\bar{T}[\bar{a}]$ in the sense that it does not depend up on the choice of $\bar{a}$.

Let $R$ be an artinian local ring such that $\bar{R}$ is an absolutely algebraic field. Suppose that $f_{1}(x), f_{2}(x) \in P[x]$ are partially irreducible polynomials over $P$ such that $\overline{f_{1}}(x)=\overline{f_{2}}(x)$. Let $\bar{a} \in \bar{R}$ such that $\overline{f_{2}}(\bar{a})=\bar{f}_{1}(\bar{a})=0$. Then $\bar{a}$ has a lift algebraic element $a_{1} \in R$ over $P$ such that $f_{1}\left(a_{1}\right)=0$. So a lifting subring $P\left[a_{1}\right]$ of $\bar{P}[\bar{a}]$ is determined by the polynomial $\bar{f}_{1}(x)$ in the sense that it does not depend up on the choice of $f_{i}(x) \in P[x], i=1,2$.

Let $R$ be a commutative local ring such that $\bar{R}$ is an absolutely algebraic field, $J(R)$ a nilpotent ideal.

(i) If char $R=0, T$ a subfield of $R$ and $b \in R$ a lift algebraic element over $P$. Then $T[b]$ is a subfield of R;

(ii) If char $R=p^{n}, T$ a Galois subring of $R$ and $b \in R$ a lift algebraic element over $P$. Then $T[b]$ is a Galois subring of $R$.

\section{Coefficient subrings}

In this section we will fucose on the ranscendental extensions of certain rings and tried to find a coefficient subring of such rings.

Definition 3 Let $T$ be a subring of a ring $R$. Then

(i) $a \in R$ is said to be an algebraic element over $T$ if it is satisfies a regular polynomial over $T$.

(ii) $a \in R$ is is said to be a transcendental element over $T$ if it is not algebraic element over $T$.

It is easy to see that if $R$ is an artinian local ring such that $\bar{R}$ is a field, $a \in R$ is an algebraic element over $P$, then there exists a monic polynomial $f(x) \in P[x]$ such that $f(a)=0$.

Lemma 4 Let $R$ be a local ring with $J(R)$ a nil ideal such that $\bar{R}$ is a field. Then $a \in R$ is a transcendental element over $\mathrm{P} \subseteq R$ if and only if $\bar{a}$ is a transcendental element over $\overline{\mathrm{P}} \subseteq \bar{R}$.

The lifting of an algebraic element over a residue field for a certain ring is a root of a lifting of the irreducible polynomial of that element, the uniqueness of the lifting element in a Hensel ring is the key point to show the existence of a coefficient subring, however a lifting of transcendental element is not a root for any polynomial, in the following proposition we will use the fact that for a transcendental element a, $a+\langle f(a)\rangle, f(a) \in P[a]$ is an algebraic element over $P$ and hence we can use Hensel lemma.

Proposition 5 Let $R$ be a local ring such that for some $\theta \in R, \theta R=R \theta$ is a minimal ideal of $R, R / \theta R$ is a commutative ring of characteristic $p^{n}, J(R)=p R+\theta R$ and $p R \cap \theta R=0$.

Let $a \in R \backslash J(R)$ such that for some $h \neq 0$ in $\theta R, a h=h a$. Then $a \in C(R)$.

Proof: Let $S=\{y \in \theta R \mid a y=y a\}$. Let $z \in S, r \in R$. Then $a z r=z a r=z \bar{a} r=z r a$. Hence $S=\theta R$. Let $x \in J(R)$. Then $x=p r+\theta s, a x=p a r+a \theta s$. At the same time $a r-r a \in \theta R$ and $p \theta=0$, therefore par $=$ pra. Hence $a x=x a$. Let $b \in R$ be a unit. Then $a b-b a=\eta=\theta s$ for some $s \in R, b^{-1} a b=a+\theta t$ for some $t \in R$. Hence $a\left(b^{-1} a b\right)=a(a+\theta t)=(a+\theta t) a$. Thus $T=P\left[a, b^{-1} a b\right]=P[a][\lambda]$, where 
$\lambda=\theta t$. Observe that $\langle p, f(a)\rangle$ in $P[a]$ is a maximal ideal, so $\frac{P[a]}{\langle p, f(a)\rangle}$ is a field.

Case 1. $a$ is lift algebraic element over $P$. Let $f(x)$ be a minimal polynomial of $a$ over $P$. This means $f(x)$ is monic, $f(x)$ is irreducible modulo $J(P)$ and $f(a)=0$ is a root. As $b^{-1} a b$ is also a root of $f(x)$ and it equals $a$ modulo $J(T)$, therefore $a=b^{-1} a b$. Hence $a b=b a$.

case 2. $a$ is not lift algebraic. Suppose $\lambda \neq 0$. As $P$ is special primary ring, $g(a) \neq 0$ for any $0 \neq g(x) \in P[x]$. Thus we have natural mapping $\tau: P[x][y] \rightarrow T$ with $\tau(x)=a, \tau(y)=\lambda$ with $y^{2}=0$. It is monic on $P[x] . \frac{T}{T \cap J(R)} \cong \bar{P}[\bar{a}]$, which is a PID, $p P[a]+\lambda P[a] \subseteq T \cap J(R)$ and $\frac{T}{p P[a]+\lambda P[a]}$ is a natural image of $P[a]$, which shows that $p P[a]+\lambda P[a]=T \cap J(R)$. As $\operatorname{ann}_{T}(\lambda)=T \cap J(T)$. Thus $\lambda T \cong \frac{T}{T \cap J(R)}$, which is not simple. Similarly, $P[a] \cap J(R)=P[a] \cap T=p P[a]$. Let $f(x) \in P[x]$ be any monic irreducible polynomial irreducible modulo $p$. Then $\langle p, f(x)\rangle$ is a maximal ideal of $P[x]$. Now $\frac{P[x, y]}{\langle p, f(x), y\rangle} \cong \frac{F[x]}{\langle p, f(x)\rangle}$,

which is a field, hence $\langle p, f(x), y\rangle$ is a maximal ideal of $P[x, y]$. Under $\tau$ its image is $M=\langle p, f(a), \lambda\rangle$. As $y^{2}=0, \frac{P[x, y]}{\langle p, f(x)\rangle}$ is a chain ring of composition length 2 , then $\frac{\langle p, f(x)\rangle}{\langle f(x)\rangle}$ is nilpotent. Hence $\frac{P[x, y]}{\langle f(x)\rangle}$ is a Hensel ring with maximal ideal $\frac{\langle p, f(x), y\rangle}{\langle f(x)\rangle}$. By using $\tau$, we get $\bar{T}=\frac{T}{\langle f(a)\rangle}$ is a Hensel ring with maximal ideal $\frac{\langle p, f(a), \lambda\rangle}{\langle f(a)\rangle}$ As $a, b^{-1} a b$ both are roots of same irreducible polynomial, so they are equal modulo $\langle f(a)\rangle$. Hence $\lambda=a-b^{-1} a b=f(a)(g(a)+\lambda h(a))$. Then $f(a) g(a) \in P[a] \cap J(R)=p P[a], g(a)=p w(a)$ for some $w(a) \in P[a]$ as $f(a)$ is not a zero divisor modulo $p P[a]$, Then $\eta(1-f(a) h(a))=p f(a) w(a)$. If $1-f(a) h(a) \in p P[a]$, then $f(a)$ is a unit in $P$, which is a contradiction. Hence $1-f(a) g(a)$ is not a zero divisor in $P[a]$. Thus $\lambda(1-f(a) g(a)) \neq 0$. As $\theta R$ is simple, $\lambda(1-f(a) g(a)) R=\theta R$. This shows that $\theta R \cap p R \neq 0, \theta R \subseteq p R$. This gives a contradiction. Hence $\lambda=0$.

The following example shows that the above result will not be true if $\theta R \subseteq p R$.

Example 6 Let $S=Z_{p 2}, R^{\prime}=S[x, y]$ such that $x y-y x=p, x y=y x+p$. Then as $p^{2}=0$, we have $x^{m} y^{k}=y^{k} x^{m}+m k p y^{k-1} x^{m-1}$. Thus, if $p$ divides $m$ or $k$, then $x^{m} y^{k}=y^{k} x^{m}, f(x, y) \in p S[x, y]$ is regular. $p(x y-y x)=p^{2}=0$, implies that $p x y=p y x$, and hence $p f g=p g f$, for any $f, g \in R^{\prime}$. Now $p R^{\prime}$ being isomorphic to $\frac{R^{\prime}}{p R^{\prime}}$ is noetherian. So $R^{\prime}$ is noetherian.

Let $G$ be the set of all regular elements of $R^{\prime}$. Let $f, g \in R^{\prime}$ with $g \neq 0$. Then 
$f g=g f+p c$ for some $c \in R^{\prime}$.

Suppose $f \in G$, and $g$ is also regular. Then

$f g g=g f g+p c g=g f g+p g c, f u=g v$,

where $u=g^{2} \in R^{\prime}, v=f g+p c$ is regular. Suppose that $g \in p R^{\prime}$. Then $g=p g^{\prime}$ for some $g^{\prime} \in G$.

$$
f g g^{\prime}=g f g^{\prime}+p c g^{\prime}=g f g^{\prime}+p g^{\prime} c=g f g^{\prime}+g c=g\left(f g^{\prime}+c\right) \text {. }
$$

At the same time $f g g^{\prime}=p f g^{\prime 2} \neq 0$. Therefore $g\left(f g^{\prime}+c\right) \neq 0$. Hence $f g^{\prime}+c \in G$. This proves that $R^{\prime}$ satisfiesÂ... Ore condition on the right. Hence we have $R$, the right localization of $R^{\prime}$ at $G$. Then $(p R)^{2}=0$ and any element of $R$ outside $p R$ is invertible. Thus $R$ is a local ring and $p R$ is its minimal ideal. Here $R$ is non-commutative, $\frac{R}{p R}$ is of characteristic $p, p R=R(p I), R / p R$ is commutative and we can assume $\theta=p I$.

It is clear that if in the above proposition we assume that $R / \theta R$ is a field then the proposition will be also true.

Proposition 7 Let $R$ be a local ring such that for some $\theta \in R, \theta R=R \theta$ is a minimal ideal of $R$, $R / \theta R$ a commutative ring of characteristic $p^{n}$ and $J(R)=p R+\theta R, p R \cap \theta R=0$. Suppose that $a, b \in R \backslash J(R)$. Then there exists $h \in \theta R$ such that

$$
a(b+h)=(b+h) a .
$$

Proof : Let $a \theta=\theta a^{\prime}$ for some $a^{\prime} \in R$. Suppose such an $h$ exist. Then $h=\theta t, a b-b a=h a-a h=\theta t a-a \theta t=\theta t a-\theta a^{\prime} t=h\left(a-a^{\prime}\right)$. If $a-a^{\prime}$ is a unit, we can take $h=(a b-b a)\left(a-a^{\prime}\right)$. Suppose $a-a^{\prime}$ is a non-unit. Then

$\theta\left(a-a^{\prime}\right)=0, \theta a=a \theta$.

By Proposition 5, $a \in C(R)$, therefore, we may take $h=0$.

Definition $8 A$ subring [subfield ] $T$ of a ring $R$ is called a coefficient subring [subfield] if $\bar{T}=(T+J(R)) / J(R)=\bar{R}$ i.e., $R=T+J(R)$ and $J(T)=T \cap J(R)=q T$, where $q=$ char $\bar{R}$. Definition 9 Let $R$ be a local ring with $J(R)$ a nil ideal. Then $R$ is called an absolutely algebraic ring if every element in $R$ is algebraic over the prime subring $P$ of $R$, otherwise $R$ is called a transcendental extension.

Let $R$ be a local ring with $J(R)$ a nil ideal such that $\bar{R}$ is a field. Let $K$ be the set of all algebraic elements in $R$ over $P$. it is clear that $a-b, a b \in K$ for any algebraic elements $a, b \in R$ over $P$. Hence $K$ is an absolutely algebraic ring. As $J(R)$ is a nil ideal, $J(R) \subseteq K$. Since $\bar{K}=K / J(R)$ is an absolutely algebraic subfield of $\bar{R}$. Then $K$ is a local ring with $J(K)=J(R)$ a nil ideal.

Theorem 10 [2] Let $R$ be an artinian local duo ring such that char $R=0$ or $p^{n}$ and $\bar{R}$ be an absolutely algebraic field. Then $R$ has a coefficient subring.

Moreover any coefficient subring of $R$ is a generalized Galois ring and any two coefficient subrings of $R$ are isomorphic.

Let $R$ be an artinian local duo ring with $d(R)=2$. Then we have the following proposition which we will need it later. 
Proposition 11 Let $R$ be a local duo ring with $d(R)=2$ and $S$ a commutative subring of $R$, let, $b, x, y \in R$ be three units elements such that $b, x \in S, y \notin S$ such that $x y=y x, b(y+h)=(y+h) b$ for some $h \in \theta R$. If $h \neq 0$ then either $x$ or $b \in C(R)$.

Proof : Here $x b=b x, x y=y x$. As $d(R)=2$, clearly, $J(R)=p R+\theta R$, where $p=\operatorname{ch}\left(\frac{R}{J(R)}\right)$. Now $b y-y b \in \theta R$, therefore $b y=y b+t, t \in \theta R$. Thus $b y=y_{1} b$, where $y_{1}=y+h^{\prime}$ for some $h^{\prime} \in \theta R$. Now $x y_{1} b=x b y=b x y=b y x=y_{1} b x=y_{1} x b$, hence $x y_{1}=y_{1} x, h^{\prime} x=x h^{\prime}$. Thus, if $h^{\prime} \neq 0$, then $x 2 C(R)$. Suppose $h^{\prime}=0$. Then so $b y=y b, b h=h b$. In this case $b \in C(R)$.

Remark 12 Let $R$ be a local duo ring with $J(R)$ a nilpotent ideal, and $P$ its prime subring. Let $a, b \in R$ be algebraic elements over $P$. Then there exists a regular polynomial $f(x) \in P[x]$ such that $f(a)=0$, hence $\bar{f}(a)=\overline{0} \cdot \bar{f}(x)$ is a non-zero polynomial over the fiÂ...eld $\bar{P}$. Thus we can fiÂ...nd a monic polynomial of smallest degree of $\bar{a}$ say $\bar{g}(x)$ over $\bar{P}$ such that $\bar{g}(\bar{a})=\overline{0}$. Now $\bar{a}-b$ is algebraic over $\bar{P}$. Let $h(x) \in P[x]$ be a monic polynomial such that $\bar{h}(\overline{a-b})=\overline{0}$. Then $h(a-b) \in J(R), h(a-b) s=0$ for some $s>0$. Hence $a-b$ is algebraic over $P$. Similarly, $a b$ is algebraic over $P$. Hence $H$ the set of all those elements of $R$ that are algebraic over $P$ is a subring of $R$. Now $H \cap J(R) \subseteq J(H)$. Let $a \in H / J(H)$, then $a$ is a unit in $R$ and ${ }^{\bar{a}}-1$ is algebraic over $\bar{P}$, which gives that $a^{-1}$ is algebraic over $P$. This proves that $H$ is a local ring with $J(H)=H \cap J(R)$. Let $L$ be the set of all lift algebraic elements in $R$. Clearly $L \subseteq H$. Let $a, b \in L$. Then $\bar{P}[\bar{a}, \bar{b}]=\bar{P}[\bar{c}]$ for some $c \in P$ which is lift algebraic over $P$. Now $\bar{P}[\bar{c}]$ is a $\hat{A}$...field or a Galois ring. As $\bar{P}[\bar{a}] \subseteq \bar{P}[\bar{c}]$, so $P[a] \subseteq P[c]$. Similarly $P[b] \subseteq P[c]$. Then $K=P[L]$ is a fiÂ...eld or a generalized Galois ring such that $H=K+J(H)$, and $K=\bigcup_{i=1}^{\infty} P\left[a_{i}\right]$, with $P\left[a_{i}\right] \subseteq P\left[a_{i+1}\right]$. Let $\alpha \in R$ be a transcendental element over $P$. We want find for some $z \in J(R), \alpha+z$ commutes with every $a_{i}$, if $J(R)=p R+\theta R, p R \cap \theta R=0$, then by Proposition 6, there exists $h_{i} \in J(R)$ such that $a_{i}\left(\alpha+h_{i}\right)=\left(\alpha+h_{i}\right) a_{i}$.

Suppose that $h_{n} \neq h_{n+1}$. Then as $P\left[a_{n}\right] \subseteq P\left[a_{n+1}\right], \alpha+h_{n+1}$ commutes with $a_{n}$. Then $a_{n} h=0$, where $h=h_{n+1}-h_{n}$, and hence $a_{n} \in C(R)$. Thus, if for any $n$, there exists an $m>n$ such that $h_{m} \neq h_{m+1}$, then $K \subseteq C(R)$, in this case we can choose $h=0$. Otherwise, there exists an $n$ such that $h_{m}=h_{n}$ for every $m>n$. Then $h=h_{n}$. Thus we can take $\alpha$ such that $\alpha$ commutes with every $a_{i}$. We get a commutative subring $K[\alpha]$. We can localize it at its regular elements and get $K_{1}$, as $K$ is a union of an ascending chain of subrings each of which is generated by an element over $P$. Suppose that we have a transcendental element $\beta$ which is algebraically independent from $\alpha$ over $P$. To get analogous commutative subring $K_{1}[\beta]$, we aim to find an element, say $\beta$ commuting with all $a_{i}, \alpha$.

Now, if $J(R)=p R+\theta R, p R \cap \theta R=0$ then by Proposition 7, there exists $h \in J(R)$ such that $\alpha(\beta+h)=(\beta+h) \alpha$.

Let $\beta^{\prime}=(\beta+h)$. Then $\alpha \beta^{\prime}=\beta^{\prime} \alpha$ and as in above remark there is $h \in J(R)$ such that $\beta^{\prime}+h$ commutes with every $a_{i}$. Now, for $d(R)=2$ we can use Proposition 11 and get, if $h \neq 0$ then either $\alpha$ or $a_{i} \in C(R)$ for 
all $i$. Therefore $K_{1}\left[\beta^{\prime}\right]$ is a commutative subring of $R$.

Theorem 13 Let $\mathrm{R}$ be a transcendental extension local duo ring with $\operatorname{char} R=0, d(R)=2$ and $\bar{R}$ is a field. Then $R$ has a coefficient subfield.

Proof: Let $K$ be the subring of all algebraic elements in $R$ over its prime subring $P$. We can find a subfield $H=\bigcup_{i=1}^{\infty} P\left[a_{i}\right]$ of $K$ with each $a_{i}$ an algebraic element over $P=\mathrm{Q}$ such that

$$
K=H+J(R) .
$$

Suppose that $\alpha \in R$ is a transcendental element over $P$. Then there exists $h \in J(R)$ such that $H[\alpha+h]$ is a commutative subring of $R$. As $\alpha$ is transcendental over $H, \alpha+h$ is also transcendental over $H$. So we can find $H\left[\alpha_{i}\right]_{i}$, such that each $\alpha_{i}$ is transcendental and $\bar{R}=\overline{H\left[\alpha_{i}\right]_{i}}$, so $H\left[\alpha_{i}\right]_{i}$ is an integral domain. Since $R$ is a local ring, $R$ contains the inverses of all non-zero elements in $H\left[\alpha_{i}\right]_{i}$. So $R$ contains the quotient field $H\left(\alpha_{i}\right)_{i}$ of $H\left[\alpha_{i}\right]_{i}$. Now

$$
R=H\left(\alpha_{i}\right)_{i}+J(R)
$$

Thus $H\left(\alpha_{i}\right)_{i}$ is a coefficient subfield of $R$.

Theorem 14 Let $\mathrm{R}$ be a transcendental extension artinian local duo ring with char $R=0$ and $\bar{R}$ is a field. Then $R$ has a coefficient subfield.

Proof : We will prove the result by induction on $d(R)$. Let $d(R)=2$. Then by Theorem $13, R$ has a coefficient subfield. Suppose that for $d(R) \leq m-1, m \geq 3, R$ has a coefficient subfield. Let $d(R)=m, R^{\prime}=R / \theta R$, where $\theta R$ is a minimal right ideal of $R$. Let $K$ be the subring of all algebraic elements in $R$ over $P$ and suppose that $\bar{R}=\overline{K\left(\alpha_{i}\right)_{i}}$, for some transcendental elements $\alpha_{i}$ in $R$ over $P$. As $R$ is an artinian local duo ring, $R^{\prime}$ is an artinian local duo ring with $d\left(R^{\prime}\right)=m-1$. So $R^{\prime}$ has a coefficient subfield, say $T / \theta R$, where $T$ is a subring of $R$ containing some $\alpha_{i}^{\prime} \in \bar{\alpha}_{i}$. Since $T / \theta R$ is a field, $J(T)=\theta R$ and hence

$$
\begin{aligned}
& R / \theta R=T / \theta R+J(R / \theta R) \\
& =T / \theta R+J(R) / \theta R .
\end{aligned}
$$

So

$$
R=T+J(R)
$$

Thus

$$
\theta R=\theta T, R \theta=T \theta \text {. }
$$

Therefore $\theta T=T \theta$ is a minimal ideal of $T$ and hence $T$ is an artinian local duo ring such that $J^{2}(T)=0$. So $d(T)=2$. By Proposition 13, $T$ has a coefficient subfield, say $F$. Now

$$
\begin{aligned}
& R=T+J(R) \\
& =F+J(T)+J(R)
\end{aligned}
$$




$$
=F+J(R) .
$$

Hence $F$ is a coefficient subfield of $R$.

Proposition 15 Let $\mathrm{R}$ be a transcendental extension local duo ring with char $R=p^{n}, d(R)=2$ and $\bar{R}$ a field. Then $R$ has a coefficient subring.

Proof: Let $K$ be the subring of all algebraic elements in $R$ over $P$, we can find a generalized Galois ring $K_{0}=\cup_{i=1}^{\infty} P\left[a_{i}\right]$ such that $K=K_{0}+J(R)$. Suppose that $\bar{R}=\overline{K\left(\alpha_{i}\right)_{i}}$, for some transcendental element $\alpha_{i}$ in $R$ over $P$. So there exists an element $h_{i} \in J(R)$ such that $K_{0}\left[\alpha_{i}+h_{i}\right]_{i}$ is a commutative subring of $R$. Now $K_{0}$ is a special primary ring. Since $R$ is an artinian local ring, the non-zero divisor elements in $K_{0}\left[\alpha_{i}+h_{i}\right]_{i}$ are units in $R . R$ contains the total qoutient ring of $K_{0}\left[\alpha_{i}+h_{i}\right]_{i}$, say $R_{0}=\left(K_{0}\left[\alpha_{i}+h_{i}\right]_{i}\right)_{\mathrm{P}}$, where $\mathrm{P}=p K_{0}\left[\alpha_{i}+h_{i}\right]_{i}$. Now $J\left(R_{0}\right)=R_{0} \cap J(R)=p R_{0}$ and $R=R_{0}+J(R)$. Thus $R_{0}$ is a coefficient subring of $R$.

Theorem 16 (The main Theorem) Let $\mathrm{R}$ be a transcendental extension artinian local duo ring with char $R=p^{n}$ and $\bar{R}$ is a field. Then $R$ has a coefficient subring.

Proof: We will prove the result by induction on $d(R)$. Let $d(R)=2$. Then by Proposition 13 , $R$ has a coefficient subring. Suppose that for $d(R)=m-1, m \geq 3, R$ has a coefficient subring. Let $d(R)=m$. Let $R^{\prime}=R / N$, where $N$ is a minimal right ideal of $R$. Then $N=\theta R$ for some $\theta \in R$. Let $K$ be the subring of all algebraic elements in $R$ over $P$ and suppose that $\bar{R}=\overline{K\left(\alpha_{i}\right)_{i}}$, for some transcendental elements $\alpha_{i} \in R$. Now $R^{\prime}$ is an artinian local duo ring with char $R^{\prime}=p^{n^{\prime}}$, where $n^{\prime} \leq n$ and $d\left(R^{\prime}\right)=m-1$. Hence $R^{\prime}$ has a coefficient subring, say $T / N$. Therefore $R=T+J(R)$. As $J(T / N)=p(T / N)$, Since $R \theta$ is a minimal ideal, $\theta R=\theta T$ and $R \theta=T \theta$. So $\theta T=T \theta$ is a minimal ideal of $T, \bar{T}=\bar{R}$ is a field. Thus $T$ is a local ring with $J(T)=T \cap J(R)$ a nilpotent ideal. Let $\bar{P}=(P+J(T)) / J(T)$ and consider an element $a+J(T) \in \bar{T} \backslash \bar{P}$ algebraic over $\bar{P}$. Then there exists a lift algebraic element $a^{\prime} \in a+J(T)$ in $T$ over $P$ such that $P\left[a^{\prime}\right]$ is a Galois subring of $R$. If $b+J(T) \in \bar{T} \backslash \bar{P}[a+J(T)]$ is an algebraic element over $\bar{P}$, then there exist an element $b^{\prime} \in b+J(T)$ in $T$ such that $L=P\left[a^{\prime}, b^{\prime}\right]$ is a Galois subring of $T$. As $J(K)=J(R)$,

$$
\bar{T} \cong \overline{K\left(\alpha_{i}\right)_{i}}
$$

Since $\alpha_{i}$ is a transcendental element over $P, \alpha_{i}^{\prime}+J(T)$ is a transcendental element over $\bar{P}$. As $\bar{K}$ is an absolutely algebraic field,

$$
\bar{T}=\left(\bigcup_{j=1}^{\infty} \bar{P}\left[a_{j}+J(T)\right]\right)\left(\alpha_{i}^{\prime}+J(T)\right)_{i},
$$

for each $j, a_{j}+J(T)$ is an algebraic element over $\bar{P}$. Now $P\left[a_{1}\right]$ contains a lift algebraic element $b_{1}$ such that $\overline{b_{1}}=\overline{a_{1}}$. Suppose for some $n, 1 \leq n<\infty$, we found a lift algebraic elements $b_{1}, \cdots, b_{n}, b_{j} \in \overline{a_{j}}$ such that $P\left[b_{j}\right] \subseteq P\left[b_{j+1}\right]$ for $1 \leq j \leq n-1$. Put $T_{n}=P\left[b_{n}\right]$. Then $\bar{P}\left[a_{n}+J(T)\right]=\overline{T_{n}}=\left(T_{n}+J(T)\right) / J(T)$. Since

$$
\bar{P}\left[a_{n}+J(T)\right] \subseteq \bar{P}\left[a_{n+1}+J(T)\right],
$$


then as seen above there exists $c_{n+1} \in T$ such that $P\left[b_{n}, c_{n+1}\right]$ is a Galois subring of $T$, $c_{n+1}+J(T)=a_{n+1}+J(T)$. So $P\left[b_{n}, c_{n+1}\right]=P\left[b_{n+1}\right]$ for some lift algebraic element $b_{n+1} \in T$ over $P$. Put $T_{n+1}=P\left[b_{n+1}\right]$. Then

$$
\bar{P}\left[a_{n+1}+J(T)\right]=\overline{T_{n+1}},
$$

and $T_{n} \subseteq T_{n+1}$. Hence by induction, we get ascending chain $\left\{T_{n}\right\}_{n=1}^{\infty}$ of Galois subrings of $T$ such that

$$
\overline{T_{n}}=\bar{P}\left[a_{n}+J(T)\right],
$$

for every $n$. Let $T_{0}=\cup_{n=1}^{\infty} T_{n}$. Then $T_{0}$ is a generalized Galois subring of $T$ and there exists $h_{i} \in \theta T$ such that $T_{0}\left[\alpha_{i}^{\prime}+h_{i}\right]_{i}$ is a commutative subring of $T$. Thus $T$ contains the ring of quotients $R_{0}$ of $T_{0}\left[\alpha_{i}^{\prime}+h_{i}\right]_{i}$ with respect to $\mathrm{P}=p T_{0}\left[\alpha_{i}^{\prime}+h_{i}\right]_{i}$. Now $R_{0}=T_{0}\left(\left[\alpha_{i}^{\prime}+h_{i}\right]_{i}\right)_{\mathrm{P}}, J\left(R_{0}\right)=p R_{0}=R_{0} \cap J(T) . T=R_{0}+J(T)$. Now $R=R_{0}+J(R), J\left(R_{0}\right)=R_{0} \cap J(R)$. Thus $R_{0}$ is a coefficient subring of $R$.

Theorem 17 Let $\mathrm{R}$ be a transcendental extension artinian local duo ring with char $R=p^{n}$ and $\bar{R}$ is a field. Then any coefficient subring of $R$ is a transcendental extension of a generalized Galois subring. Moreover any two coefficient subrings of $R$ are isomorphic.

Proof: Let $T$ be coefficient subring of $R$. Then $R=T+J(R), J(T)=J(R) \cap T=p T$ and hence $\bar{T} \cong \bar{R}$ is a transcendental field extension of an absolutely algebraic field. So $T$ has a finite composition length and all one sided ideals are of the form $p^{i} T, 0 \leq i \leq n$. Then $T$ is an artinion duo ring and hence it has a coefficient subring $T_{0}$ which is a transcendental extension of a generalized Galois subring. We can easly see that $T=T_{0}$.a transcendental extension of a generalized Galois subring in $R$. Moreover, let us suppose that $T=K\left(\left[\alpha_{i}\right]_{i}\right)_{\mathrm{P}}$ and $T^{\prime}=K^{\prime}\left(\left[\alpha_{i}^{\prime}\right]_{i}\right)_{\mathrm{P}^{\prime}}$ are two coefficient subrings of $R$, we can choose $\alpha_{i}^{\prime}$ such that $\overline{\alpha_{i}}=\overline{\alpha_{i}^{\prime}}$ transcendental elememts and $K, K^{\prime}$ are generalized Galois subrings of $R$.. Then it is clear that $\bar{K} \cong \overline{K^{\prime}}$ and from [2], we get $K \cong K^{\prime}$. Let $\eta: K \rightarrow K^{\prime}$ be an isomorphism. So we get an isomorphism $\eta^{\prime}: K\left[\alpha_{i}\right]_{i} \rightarrow K^{\prime}\left[\alpha_{i}^{\prime}\right]_{i}$ that extends $\eta$, such that $\eta^{\prime}\left(\alpha_{i}\right)=\alpha_{i}^{\prime}$, for all $i$. Let $\mathrm{P}=p K\left[\alpha_{i}\right]_{i}$. Then $\eta\left(p K\left[\alpha_{i}\right]_{i}\right)=p K^{\prime}\left[\alpha_{i}^{\prime}\right]_{i}=\mathrm{P}^{\prime}$ and $\eta^{\prime}$ extends to the isomorphism $\overline{\eta^{\prime}}: K\left(\left[\alpha_{i}\right] i\right)_{\mathrm{P}} \rightarrow K\left(\left[\alpha_{i}^{\prime}\right]_{i}\right)_{\mathrm{P}^{\prime}}$. This proves the result.

\section{Acknowledgement}

The author would like to thank Professor Surjeet Singh for a correspondence regarding this work as well as for providing his valued remarks.

\section{References}

[1] Alkhamees, Y., Singh, S. 2002. Internal subrings of a local finite algebra. Colloquium Mathematicum. 92(2002), 35-43.

[2] Alkhamees, Y., Alolaiyan, H., Singh, S. 2003. A representation theorem for chain rings. Colloquium Mathematicum. 96(2003), 103-119.

[3] Azarang,A. 2015. On the existence of maximal subrings in commutative noetherian rings. Journal of Algebra and Its Applications. Vol. 14, 1(2015), $196 a ̂ € " 207$.

[4] Azumaya, G. 1951. On maximally central algebras. Nagoya Math. J. 2 (1951), 119-150.

[5] Azumaya, T. 1995. Coefficient subrings of certain local rings with prime-power characteristic. International Journal of Mathematics and Mathematical Sciences. 18 (3) (1995), 451-462. 


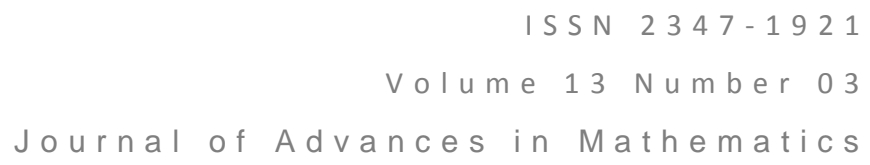

[6] Clark, W. E. 1972. A coefficient ring for finite non-commutative rings. Proc. Amer.Math.Soc. 51 (1972), $25-28$.

[7] Cohen, I. S. 1946. On the structure and ideal theory of complete local rings. Trans. Amer. Math. Soc. 59 (1946), 54-28.

[8] Corbas, B. A coefficient subring of finite rings. unpublished notes.

[9] Faith, C. 1976. Algebra II, Ring Theory. Grundlehren Math. Wiss. 191, Springer.

[10] Gilmer, R. 1972. Multiplicative Ideal Theory. Pure Appl. Math. 12, Dekker.

[11] Krull, W. 1924.Algebraische theoie der ring. 11, Math. Ann. 91(1924), 1-46.

[12] McConnell, J. C. Robson, J. C. 1987. Noncommutative Noetherian Rings. Wiley.

[13] Ã-inert, J. Richter, J. Silvestrov, S. D. 2013. Maximal commutative subrings and simplicity of Ore extensions. Journal of Algebra and Its Applications. 12 (4)(2013).

[14] Pavelescu, A. 2013. Some Maximal Commutative Subrings of M n (D). Communications in Algebra. 41(2013), 3032â€"3034.

[15] Pierce, R. S. 1982. Associative Algebras. Grad. Texts in Math. 88, Springer.

[16] Richter, J. Silvestrov, S. D. 2014. Centralizers in Ore extensions of polynomial rings. International Electronic Journal of Algebra. Vol. 15 (2014), 196â€"207.

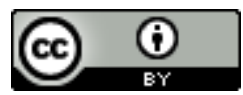

This work is licensed under a Creative Commons Attribution 4.0 International License. 